\title{
Overexpression of NLRPs as Independent Indicator for Prognosis of Patients with Head and Neck Squamous Cell Carcinoma
}

\section{Jiayuan Xu}

the Third Affiliated Hospital of Wenzhou Medical School

\section{Li Bangliang}

the Third Affiliated Hospital of Wenzhou Medical School

\section{Wang Wen}

Wenzhou Medical College: Wenzhou Medical University

S Lin ( $\nabla$ Inns@163.com)

the third affiliated hospital of Wenzhou Medical University https://orcid.org/0000-0002-1175-0232

\section{Research}

Keywords: NOD-like receptor proteins, head and neck squamous cell carcinoma, metastasis; survival, NLRPs, miRNAs, HNSCC

Posted Date: July 29th, 2021

DOI: https://doi.org/10.21203/rs.3.rs-585851/v2

License: (9) This work is licensed under a Creative Commons Attribution 4.0 International License. Read Full License 


\section{Abstract}

NOD-like receptor proteins (NLRPs) are an important part of gene regulation and play a vital role in many cancers. Recently, some studies have shown that members of the NLRP family are a crucial component in the progression of head and neck squamous cell carcinoma (HNSCC) and can be used as the main therapeutic targets. To explore this hypothesis, bioinformatics analyses using Gene Expression Profiling Interactive Analysis (GEPIA), Oncomine, UALCAN, the Human Protein Atlas, and the cBioPortal database were performed in this study to determine the intracellular roles and functions of NLRPs. String and Cytoscape were also employed to investigate and visualize the proteins in intracellular pathways related to the regulation of HNSCC carcinogenesis. Further analysis revealed that the overexpression of NLRPs was significantly related to the HNSCC stage and the survival of patients. We also explored the miRNAs downstream of NLRPs involved in nasopharyngeal carcinoma using GEO2R, TargetScan, and miRDB, and found that NLRPs can serve as prognostic biomarkers for the survival of HNSCC patients.

\section{Introduction}

Head and neck squamous cell carcinoma (HNSCC) is the sixth most common cancer in the world. According to data from the Global Cancer Observatory (GLOBOCAN), a 30\% increase in the incidence of HNSCC is predicted to occur by 2030 (1.08 million new cases per year)[1][2]. More remarkably, the 5-year survival rate for HNSCC ranges from $40-50 \%$ [3] and is only $34.9 \%$ for patients with advanced HNSCC[4] . Thus, obtaining an accurate diagnosis of HNSCC at the early stage and predicting the prognosis of HNSCC have become the research focus for head and neck cancer treatment under the current circumstances. In recent years, there have been many reports on the mechanisms, development, and metastasis of HNSCC, but the biomarkers and potential therapeutic targets for HNSCC remain undefined.

Members of the nucleotide-binding oligomerization domain, leucine-rich repeat, and pyrin domaincontaining (NLRP) family have been suggested to play critical roles in the carcinogenesis of HNSCC. However, though the NLRP family contains 22 members (NLRPs 1-22), we selected NLRPs 1-14 as the main focus of our research because of the limited amount of published data on NLRPs 15-22. MicroRNAs (miRNAs) are short non-coding RNAs with a length of 22-25 nucleotides that perform various posttranscriptional functions through unique complementary interactions with messenger RNAs (mRNA) [5]. In recent years, miRNA-based post-transcriptional control of NLRP3 has received substantial research attention, especially as a potential therapeutic strategy.

A comprehensive study of NLRPs can uncover the molecular mechanisms related to the oncogenesis and metastasis of HNSCC and may reveal potential targets for the treatment of HNSCC. In the present study, we analyzed the relevant evidence using bioinformatics methods to investigate the expression of NLRPs in HNSCC patients and their relationships with clinical indicators. In addition, we predicted and analyzed 
the neighboring genes for the NLRP family. Finally, we examined the miRNAs downstream of NLRPs involved in nasopharyngeal carcinoma (NPC), providing a research foundation for better treatment of NPC.

\title{
Materials And Methods
}

\author{
Microarray data
}

The Gene Expression Profiling Interactive Analysis (GEPIA) server [6][7] is a web-based tool that facilitates data mining on the RNA sequencing expression data repository. The resource was constructed using samples from the Cancer Genome Atlas (TCGA) and Genotype-Tissue Expression (GTEx) databases and covers 33 different cancers. We used GEPIA to obtain the expression levels of 12 NLRPs in different cancer tissues and the prognostic value of NLRP expression.

\section{Data processing}

The Oncomine database (www.oncomine.org)[7] integrates RNA and DNA-seq data from the Gene Expression Omnibus (GEO) and TCGA with published literature sources to provide cancer genetic information. Oncomine is usually used to identify new biomarkers. We performed differential expression analysis and co-expression analysis with Oncomine to find genes that are differentially expressed in certain cancers with the goal of identifying target genes and guiding future research.

UALCAN (http://ualcan.path.uab.edu/index.html) [8] uses TCGA level 3 RNA-seq and clinical data from 31 cancer types to analyze the relative expression of a queried gene(s) across tumor and normal samples. The tool can also analyze various tumor sub-groups based on individual cancer stages, tumor grades, races, body weights, and other clinicopathological features; estimate the effect of gene expression levels and clinicopathological features on patient survival; and identify the top over- or under-expressed (up and down-regulated) genes in individual cancer types. This resource provides a platform for in silico validation of target genes and for identifying tumor sub-group-specific candidate biomarkers. We studied the expression levels of 12 NPRLs and the associated clinicopathologic parameters using UALCAN. $p<$ 0.05 was considered statically significant (student's t-test).

The cBio Cancer Genomics Portal (http://cbioportal.org) is an open-access resource for interactive exploration of multidimensional cancer genomics datasets. It currently provides access to data from 
more than 5,000 tumor samples from 20 cancer studies, allowing users to query datasets across genes, samples, and data types to examine different biologically and/or clinically relevant hypotheses [9]. This online tool simplifies the molecular expression profile data for cancer tissues and cell lines into easy-tounderstand genetic (gene), epigenetic, gene expression, and protein events. The website also provides graphical gene-level abstracts, network visualization analysis, survival analysis, patient-centric queries, and software programming entries. In this study, we used this online platform to analyze the genome profiles of 12 NLRPs that contained mutations based on putative copy number changes obtained from the GISTIC program and an mRNA z-score (RNASeq V2 RSEM) threshold of \pm 1 .8. For the statistical analysis, Kaplan-Meier diagrams were used to visualize the mutations in NLRP genes and their association with overall survival (OS) in HNSCC patients. The log-rank test was performed to identify the significance of the differences between the survival curves. When the $p$-value was $<0.05$, the difference was considered statistically significant.

\section{Functional and pathway enrichment analyses}

Metascape is a powerful gene function annotation analysis tool that not only performs biological pathway enrichment analysis and protein interaction network structure analysis but also presents the results in a high-quality graphical language. The data in Metascape are updated quickly (updated faster than DAVID) and cover a wide range of genes and diseases. Inside the cell, a vast number of macromolecules interact dynamically with each other at all times and it is necessary to understand these interactions. String and Cytoscape are tools that can be used to create molecular maps, that is, protein interaction networks. In this study, we effectively combined these tools to determine the protein network for NLRPs. Annotation and integration using the Gene Ontology (GO) and Kyoto Encyclopedia of Genes and Genomes (KEGG) databases were carried out with Metascape and visualization was performed with Cytoscape software. GO enrichment analysis can predict the functional roles of NLRP mutations and genes significantly associated with NLRPs, while KEGG analysis can define the pathways related to the NLRPs.

The Human Protein Atlas (https://www.proteinatlas.org) integrates various omics technologies, including antibody-based imaging, mass spectrometry-based proteomics, transcriptomics, and systems biology to map the organization and cell distribution information for 26,000 human proteins in 64 cell lines and 48 human normal tissues, as well as protein expression in 20 kinds of tumor tissues. Using this open resource, we identified tumor-type specific proteins that were differentially expressed and performed a direct comparison of protein expression in different NLRPs between normal and HNSCC tissues in humans. 
The GEO database is a gene expression database created and maintained by the National Center for Biotechnology Information (NCBI) and is used for differential analysis of expression profile chips. In the

present study, we used this tool to identify differentially expressed miRNAs (DEM) in NPC. The GSE32960 dataset includes the miRNA profile of 312 NPC specimens and 18 normal nasopharyngeal tissues. Statistically significant DEMs between NPC samples and normal samples were obtained based on the false discovery rate (FDR) with an adjusted p-value less than 0.05 and a fold change (FC) less than 2.

miRNAs in mammals can play a post-transcriptional regulatory role by binding to the 3'untranslated region (UTR) region of the transcript sequence. TargetScan[10] is a tool that specifically organizes mammalian miRNA target genes into a database based on existing analysis results. Similar to the other web-based resources, the miRNA Target Prediction Database (miRDB) provides the expression profiles for over 1000 cell lines. It also provides target data tailored for specific cell models, thereby enabling the prediction of miRNA target gene information for humans, mice, and other species through MirTarget software. In this study, we combined these two software tools to investigate the miRNAs downstream of NLRPs involved in NPC.

\section{Results}

Expression of NLRPs in patients with carcinoma

Both the transcription and translation of NLRPs were analyzed using GEPIA (http://gepia.cancer-pku.cn/), UALCAN (htttp://ualcan.path.uab.edu) and the Human Protein Atlas (http://www.proteinatlas.org) to determine the prognostic and potential therapeutic values of the NLRPs in patients with carcinoma. The expression levels and mRNA expression patterns of 14 NLRPs in 31 types of cancers were obtained from the GEPIA database and 20 types of cancers were obtained from the Oncomine, respectively (Figure 1; Table 1). Compared to normal samples, 12 NLRPs (A-K: NLRP1/2/3/4/6/7/9/10) were significantly overexpressed in primary NPC tissues $(p<0.05)$ but there was no statistical difference in the expression of NLRP11, NLRP12, NLRP13 and NLRP14 ( $>0.05)$, while there was no data of NLRP

5 and 8 (Figure 2; Table 2).

The relationship between the expression of NLRPS and the clinical manifestations of HNSCC 
We employed UALCAN (http://ualcan.path.uab.edu) and The Human Protein Atlas (https://www.proteinatlas.org) to examine the relationship between the expression of NLRPs and the clinical manifestations of HNSCC, including the cancer stage and tumor grades for each patient, and found that the mRNA expression of 12 NLRPs (NLRP1/2/3/4/6/7/9/10/11/12/14) had an obvious association with the HNSCC cancer stages. This finding demonstrated that patients with advanced cancer tend to exhibit higher NLRP expression (Figure 3, Figure 4; Table 3).

Genetic mutations in NLRPs and their prognostic value

We investigated the genetic alteration in NLRPs using cBioPortal. As shown in Figure 5, NLRP 2, NLRP 3 , NLRP 4 , NLRP 5, NLRP 7, NLRP 8 , NLRP 11 ,NLRP 12 هLRP13 and NLRP 14 were genes alterations more than $3 \%$ in HNSCC patients. Furthermore, to determine the prognostic value of NLRP expression in NPC patients, we combined cBioPortal with GEPIA to investigate the relationship between NLRP expression and the OS of patients with NPC. Figures 6 show that higher mRNA expression of NLRP $1 / 2 / 7 / 6 / 14$ was associated with shorter OS in UALCAN database. Figures 7 show that higher mRNA expression of NLRP 1/6/14 was associated with shorter OS in GEPIA database.

Prediction of functions and pathways of NLRPS

After analyzing the genetic changes in members of the NLRP family and their prognostic value for HNSCC patients, we explored the functions and pathways of the NLRPs with Metascape by constructing an integrated network of neighboring genes (Figure 7) that GO enrichment and KEGG pathway analyses in Metascape were performed to understand the intracellular biological pathways of the integrated functions of NLRPs (Figure $8 \square 9 \square 10$ ). The results showed the involvement of NLRPs in the pathways pertaining to the regulation of innate immune responses to cytosolic DNA, interleukin-1 processing, and the NLRP1 inflammasome. evidently connect to the NLRPs using String and Cytoscape (Figure 11).

Prediction of target miRNAs in nasopharyngeal carcinoma patients

After a series of studies on the role of the NLRPs in HNSCC patients, we obtained data from the NCBI-GEO dataset GSE32960 to establish the value of the NLRP family in the treatment of NPC. GSE32960 contains data for 312 NPC tissue samples and 18 normal tissue samples. Applying the cut-off criteria of $<0.05$ for 
the adjusted p-value (FDR) and FC > 1 resulted in a total of 82 DEMs that were considered statistically significant. The results are shown in a volcano map (Figure 12).

To explore the target miRNAs of NLRPs in NPC, we employed two online analysis tools, miRDB (http://ww.mirdb.org/miRDB/) and TargetScanHuman 7.2 (http://ww.targetscan.org/) for miRNA prediction. The resulting Venn diagram was used to analyze the NLRPs targeted by miRNAs. The target miRNAs related to NPC survival were identified through the above rigorous research process. We eventually determined that the NLRPs are regulated by three miRNAs: miR-199a-3p regulates the expression of NLRP1, miR-125a-5p regulates the expression of NLRP2, and miR-22 regulates the expression of NLRP3.

\section{Discussion}

In recent years, the use of traditional clinicopathological indicators for the early HNSCC diagnosis and prediction of the prognosis has been insufficient $[11 ; 12]$. Therefore, new prognostic biomarkers that fully reflect the biological characteristics of tumors and the treatment efficacy of patients with HNSCC have been under investigation. To date, many studies have been conducted to identify predictive biomarkers in HNSCC patients. These potential biomarkers are divided into single-molecule protein markers and gene markers that can be identified through high-throughput analysis and gene expression profiling[13; 14 ; 15].

NLRP are involved in the progression of a variety of cancers [16]; however, their functions in HNSCC remain unknown. Our results showed that the mRNAs and proteins of 12 NLRP family members were overexpressed in HNSCC. The overexpression was significantly associated with the cancer stage in HNSCC and was also remarkably associated with shorter OS. Additionally, the mutation and genetic alteration rates of NLRPs were higher in HNSCC patients. Analysis of the functions and pathways of NLRPs and their altered neighbor genes suggested the involvement of NLRPs in the following biological processes: defense response to other organisms, I-kappaB kinase/NF-kappaB signaling, regulation of the production of type I interferons, interleukin-1, interleukin-18, pyroptosis, NLRP3 inflammasome complex assembly, female gamete generation, response to interferon-gamma, positive regulation of organelle organization, protein processing, and positive regulation of cellular component biogenesis. KEGG analysis confirmed the functions of the NLRP1 inflammasome in the regulation of the innate immune responses to cytosolic DNA and interleukin-1 processing.

Previous studies have suggested that NLRPs play a role in various cancers. Polymorphism or mutation of NLRP1A is closely related to the progression of cancer [17]. NLRP3 plays a vital role in the 
metastasis and prognosis of NPC and other cancers [18; 19; 20]. NLRP4 (a cytosolic receptor in several tissues[21]) has recently been confirmed to not only inhibit autophagy[22; 23] but also to function in the immune response to viral infections (eg Epstein-Barr virus, human papilloma virus)[24], thereby indirectly contributing to the development of NPC. NLRP7 had been reported in previous studies as the main pathogenic gene for recurrent hydatidiform moles (RHMs) [25]. NLRP12 deficiency leads to colitis and inflammation-induced tumorigenesis in mice[26; 27].

The NLRP family is significantly related to the progression of NPC in studies. Therefore, we further explored the miRNAs downstream of NLRPs involved in NPC. The results indicated that miR-199a-3p regulates the expression of NLRP1, miR-125a-5p regulates the expression of NLRP2, and miR-22 regulates the expression of NLRP3. It has previously been demonstrated in some cancers that miR-22 targets NLRP3 during carcinogenesis[28]. miR-125a-5p reportedly targets ERBB3 and inhibits cell proliferation, cell cycle progression, and cell migration in head and neck cancers [29]. In another study, upregulated miR-125a-5p was found to enhance the metastatic ability of HNSCC cell lines [30; 31]. In addition, miR-125a-5p has been identified as an important cancer treatment target in a series of cancers, including gastric cancer[32], cervical cancer [33], and lung cancer[34]. miR-199a-3p is not only expressed abnormally in a variety of cancers but also regulates ITGA3 to inhibit metastasis in head and neck cancer [35]. In a recently published study, bioinformatics analysis established miR-199a-3p as an important target for the treatment of head and neck cancer [36].

Our research has certain limitations. First, due to the small sample size in the online databases used, the breadth of our research findings is limited. A larger sample size is needed for further research to confirm our findings and explore the role of NLRPs in HNSCC. Second, our study lacks information on the diagnostic value of NLRPs in HNSCC; therefore, further research is needed to investigate whether NLRPs can be used as diagnostic biomarkers. Finally, because we did not uncover the underlying mechanisms for NLRP in HNSCC, our research lacks depth.

\section{Conclusion}

In brief, our research results confirm that the over-expression of 12 NLRPs is associated with the cancer stage in HNSCC patients. Multivariate analysis showed that higher expression of NLRPs was significantly associated with shorter OS in HNSCC patients. This study demonstrates that the NLRP family can be prognostic biomarkers for HNSCC patients. We also explored the miRNA targets downstream of NLRPs in patients with NPC, laying the foundation for further research.

\section{Declarations}

\section{Ethics approval and consent to participate}


Not applicable

\section{Consent for publication}

Not applicable

\section{Availability of data and materials}

The datasets generated and/or analysed during the current study are available in the Oncomine database (www.oncomine.org) 『cBio Cancer Genomics Portal (http://cbioportal.org)『GEPIA (http://gepia.cancerpku.cn/)『UALCAN(http://ualcan.path.uab.edu/index.html) खmetascape (https://metascape.org/gp/index.html\#/main/step1); the Human Protein Atlas(The Human Protein Atlas) $\otimes$ GEO (https://www.ncbi.nlm.nih.gov/geo/)

\section{Competing interests}

The authors declare that they have no competing interests

\section{Funding}

Not applicable

\section{Authors' contributions}

Xu Jiayuan: Conceptualization, Methodology, Writing-Original draft.

Li Bangliang: Visualization, Investigation $₫$ Software

Lin Sen: Supervision, Writing-Reviewing and Editing

\section{Acknowledgements}

Not applicable

\section{Abbreviations}

NLRP $₫ N O D$-like receptor proteins

HNSCC Øhead and neck squamous cell carcinoma

GEPIA囚Gene Expression Profiling Interactive Analysis 
GO『Gene Ontology

KEGG『Kyoto Encyclopedia of Genes and Genomes (KEGG)

GTEx冈Genotype-Tissue Expression

miRDB囚miRNA Target Prediction Database

NCBI区National Center for Biotechnology Information

TCGA囚the Cancer Genome Atlas

DEM $₫$ differentially expressed miRNAs

miRNAs: micro-RNAs

mRNA囚messenger RNAs

UTR\ untranslated region

NPCØnasopharyngeal carcinoma

FDR®false discovery rate

RHMs $₫$ recurrent hydatidiform moles

FC囚fold change

OS₫overall survival

DFS®disease-free survival

ACC: Adrenocortical carcinoma

BRCA: Breast invasive carcinoma

CHOL: Cholangio carcinoma

DLBC: Lymphoid Neoplasm Diffuse Large B-cell Lymphoma

GBM: Glioblastoma multiforme

KICH: Kidney Chromophobe

KIRP: Kidney renal papillary cell carcinoma

LAML:Acute Myeloid Leukemia 
LGG: Brain Lower Grade Glioma

LUAD: Lung adenocarcinoma

OV: Ovarian serous cystadenocarcinoma

PCPG: Pheochromocytoma and Paraganglioma

READ: Rectum adenocarcinoma

SKCM: Skin Cutaneous Melanoma

TGCT: Testicular Germ Cell Tumors

THYM: Thymoma

UCS Uterine Carcinosarcoma

\section{References}

[1]Erratum: Global cancer statistics 2018: GLOBOCAN estimates of incidence and mortality worldwide for 36 cancers in 185 countries. \%J CA: a cancer journal for clinicians. (2020). 70(4), 313.

doi:10.3322/caac. 21609

[2] Bray, F., Ferlay, J., Soerjomataram, I., Siegel, R., Torre, L., \& Jemal, A. J. C. a. c. j. f. c. (2018). Global cancer statistics 2018: GLOBOCAN estimates of incidence and mortality worldwide for 36 cancers in 185 countries. 68(6), 394-424. doi:10.3322/caac. 21492

[3] Siegel, R., Miller, K., \& Jemal, A. J. C. a. c. j. f. c. (2016). Cancer statistics, 2016. 66(1), 7-30.

doi:10.3322/caac. 21332

[4] Leemans, C., Braakhuis, B., \& Brakenhoff, R. J. N. r. C. (2011). The molecular biology of head and neck cancer. 17(1), 9-22. doi:10.1038/nrc2982

[5] Esteller, M. J. N. r. G. (2011). Non-coding RNAs in human disease. 12(12), 861-874.

doi:10.1038/nrg3074

[6] Tang, Z., Li, C., Kang, B., Gao, G., Li, C., \& Zhang, Z. J. N. a. r. (2017). GEPIA: a web server for cancer and normal gene expression profiling and interactive analyses. 45, W98-W102. doi:10.1093/nar/gkx247

[7] Rhodes, D., Kalyana-Sundaram, S., Mahavisno, V., Varambally, R., Yu, J., Briggs, B., . . Chinnaiyan, A. J. N. (2007). Oncomine 3.0: genes, pathways, and networks in a collection of 18,000 cancer gene expression profiles. 9(2), 166-180. doi:10.1593/neo.07112 
[8] Chandrashekar, D., Bashel, B., Balasubramanya, S., Creighton, C., Ponce-Rodriguez, I., Chakravarthi, B., \& Varambally, S. J. N. (2017). UALCAN: A Portal for Facilitating Tumor Subgroup Gene Expression and Survival Analyses. 19(8), 649-658. doi:10.1016/j.neo.2017.05.002

[9] Martinon, F., \& Tschopp, J. J. C. (2004). Inflammatory caspases: linking an intracellular innate immune system to autoinflammatory diseases. $117(5), 561-574$. doi:10.1016/j.cell.2004.05.004

[10] Agarwal, V., Bell, G., Nam, J., \& Bartel, D. J. e. (2015). Predicting effective microRNA target sites in mammalian mRNAs. 4. doi:10.7554/eLife.05005

[11] Gao, J., Aksoy, B., Dogrusoz, U., Dresdner, G., Gross, B., Sumer, S., . . Schultz, N. J. S. s. (2013). Integrative analysis of complex cancer genomics and clinical profiles using the cBioPortal. 6(269), pl1. doi:10.1126/scisignal.2004088

[12] Llovet, J., Ricci, S., Mazzaferro, V., Hilgard, P., Gane, E., Blanc, J., . . , J. T. N. E. j. o. m. (2008). Sorafenib in advanced hepatocellular carcinoma. 359(4), 378-390. doi:10.1056/NEJMoa0708857

[13] Cheng, A., Kang, Y., Chen, Z., Tsao, C., Qin, S., Kim, J., . . Guan, Z. J. T. L. O. (2009). Efficacy and safety of sorafenib in patients in the Asia-Pacific region with advanced hepatocellular carcinoma: a phase III randomised, double-blind, placebo-controlled trial. 10(1), 25-34. doi:10.1016/s14702045(08)70285-7

[14] Zhao, X., Sun, S., Zeng, X., \& Cui, L. J. A. j. o. c. r. (2018). Expression profiles analysis identifies a novel three-mRNA signature to predict overall survival in oral squamous cell carcinoma. 8(3), 450-461.

[15] De Cecco, L., Bossi, P., Locati, L., Canevari, S., \& Licitra, L. J. A. o. o. o. j. o. t. E. S. f. M. O. (2014). Comprehensive gene expression meta-analysis of head and neck squamous cell carcinoma microarray data defines a robust survival predictor. 25(8), 1628-1635. doi:10.1093/annonc/mdu173

[16] Tian, S., Meng, G., Zhang, W. J. C. m., \& research. (2019). A six-mRNA prognostic model to predict survival in head and neck squamous cell carcinoma. 11,131-142. doi:10.2147/cmar.S185875

[17] Girardelli, M., Maestri, I., Rinaldi, R., Tognon, M., Boldorini, R., Bovenzi, M., . . cancer. (2012). NLRP1 polymorphisms in patients with asbestos-associated mesothelioma. 7(1), 25. doi:10.1186/1750-9378-725

[18] Roberts, R., Topless, R., Phipps-Green, A., Gearry, R., Barclay, M., Merriman, T. J. G., \& immunity. (2010). Evidence of interaction of CARD8 rs2043211 with NALP3 rs35829419 in Crohn's disease. 11(4), 351-356. doi:10.1038/gene.2010.11

[19] Duewell, P., Kono, H., Rayner, K., Sirois, C., Vladimer, G., Bauernfeind, F., . . Latz, E. J. N. (2010). NLRP3 inflammasomes are required for atherogenesis and activated by cholesterol crystals. 464(7293), 13571361. doi:10.1038/nature08938 
[20] Allen, I., Wilson, J., Schneider, M., Lich, J., Roberts, R., Arthur, J., . . Ting, J. J. I. (2012). NLRP12 suppresses colon inflammation and tumorigenesis through the negative regulation of noncanonical NFKB signaling. 36(5), 742-754. doi:10.1016/j.immuni.2012.03.012

[21] Verma, D., Bivik, C., Farahani, E., Synnerstad, I., Fredrikson, M., Enerbäck, C., . . research, m. (2012). Inflammasome polymorphisms confer susceptibility to sporadic malignant melanoma. 25(4), 506-513. doi:10.1111/j.1755-148X.2012.01008.x

[22] Ahmad, I., Muneer, K., Chang, M., Nasr, H., Clay, J., Huang, C., . . photobiology. (2017). Ultraviolet Radiation-Induced Downregulation of SERCA2 Mediates Activation of NLRP3 Inflammasome in Basal Cell Carcinoma. 93(4), 1025-1033. doi:10.1111/php.12725

[23] Cui, J., Li, Y., Zhu, L., Liu, D., Songyang, Z., Wang, H., \& Wang, R. J. N. i. (2012). NLRP4 negatively regulates type I interferon signaling by targeting the kinase TBK1 for degradation via the ubiquitin ligase DTX4. 13(4), 387-395. doi:10.1038/ni.2239

[24] Eibl, C., Grigoriu, S., Hessenberger, M., Wenger, J., Puehringer, S., Pinheiro, A., . . Peti, W. J. B. (2012). Structural and functional analysis of the NLRP4 pyrin domain. 51(37), 7330-7341. doi:10.1021/bi3007059

[25] Murdoch, S., Djuric, U., Mazhar, B., Seoud, M., Khan, R., Kuick, R., . . Slim, R. J. N. g. (2006). Mutations in NALP7 cause recurrent hydatidiform moles and reproductive wastage in humans. 38(3), 300-302. doi:10.1038/ng1740

[26] Akoury, E., Gupta, N., Bagga, R., Brown, S., Déry, C., Kabra, M., . . Slim, R. J. R. b. o. (2015). Live births in women with recurrent hydatidiform mole and two NLRP7 mutations. 31(1), 120-124. doi:10.1016/j.rbmo.2015.03.011

[27] Jounai, N., Kobiyama, K., Shiina, M., Ogata, K., Ishii, K., \& Takeshita, F. J. J. o. i. (2011). NLRP4 negatively regulates autophagic processes through an association with beclin1. 186(3), 1646-1655. doi:10.4049/jimmunol.1001654

[28] Li, S., Liang, X., Ma, L., Shen, L., Li, T., Zheng, L., . . Jia, J. J. O. (2018). MiR-22 sustains NLRP3 expression and attenuates $\mathrm{H}$. pylori-induced gastric carcinogenesis. 37(7), 884-896. doi:10.1038/onc.2017.381

[29] Xu, M., Zhan, J., Xie, J., Zhu, L., Chen, L., Luo, X., . . Lu, Z. J. A., nasus, larynx. (2021). MiR-125a-5p inhibits cell proliferation, cell cycle progression, and migration while promoting apoptosis in head and neck cancers by targeting ERBB3. 48(3), 477-486. doi:10.1016/j.anl.2020.10.001

[30] Jin, S., Liu, M., Wu, H., Pang, P., Wang, S., Li, Z., . . Liu, F. J. O. I. (2018). Overexpression of hsa-miR$125 a-5 p$ enhances proliferation, migration and invasion of head and neck squamous cell carcinoma cell lines by upregulating C-C chemokine receptor type 7. 15(6), 9703-9710. doi:10.3892/ol.2018.8564 
[31] Vo, D., Karanam, N., Ding, L., Saha, D., Yordy, J., Giri, U., . . Story, M. J. N. (2019). miR-125a-5p Functions as Tumor Suppressor microRNA And Is a Marker of Locoregional Recurrence And Poor prognosis in Head And Neck Cancer. 21(9), 849-862. doi:10.1016/j.neo.2019.06.004

[32] Xiong, J., Tu, Y., Feng, Z., Li, D., Yang, Z., Huang, Q., . . therapy. (2019). Epigenetics mechanisms mediate the miR-125a/BRMS1 axis to regulate invasion and metastasis in gastric cancer. 12, 7513-7525. doi:10.2147/ott.S210376

[33] Cao, Q., Wang, N., Ren, L., Tian, J., Yang, S., \& Cheng, H. J. C. c. i. (2020). miR-125a-5p posttranscriptionally suppresses GALNT7 to inhibit proliferation and invasion in cervical cancer cells via the EGFR/PI3K/AKT pathway. 20, 117. doi:10.1186/s12935-020-01209-8

[34] Sun, C., Zeng, X., Guo, H., Wang, T., Wei, L., Zhang, Y., . . Zhang, N. J. C. b. s. A. o. D. m. (2020). MicroRNA-125a-5p modulates radioresistance in LTEP-a2 non-small cell lung cancer cells by targeting SIRT7. 27(1), 39-49. doi:10.3233/cbm-190381

[35] Koshizuka, K., Hanazawa, T., Kikkawa, N., Arai, T., Okato, A., Kurozumi, A., . . Seki, N. J. C. s. (2017). Regulation of ITGA3 by the anti-tumor miR-199 family inhibits cancer cell migration and invasion in head and neck cancer. 108(8), 1681-1692. doi:10.1111/cas.13298

[36] Liu, A., Pang, Y., Chen, G., Wu, H., He, R., Dang, Y., . . treatment. (2020). Downregulation of miR-199a$3 p$ in Hepatocellular Carcinoma and Its Relevant Molecular Mechanism via GEO, TCGA Database and In Silico Analyses. 19, 1533033820979670. doi:10.1177/1533033820979670

\section{Tables}

Due to technical limitations, Table 1 is only available as a download in the supplementary files section. 


\begin{tabular}{|ll|}
\hline NLRP family & $p$ \\
\hline NLRP1 & $1.62 \mathrm{E}-12$ \\
\hline NLRP2 & $2.01 \mathrm{E}-07$ \\
\hline NLRP3 & $2.20 \mathrm{E}-04$ \\
\hline NLRP4 & $1.43 \mathrm{E}-06$ \\
\hline NLRP5 & $/$ \\
\hline NLRP6 & $1.36 \mathrm{E}-10$ \\
\hline NLRP7 & $5.36 \mathrm{E}-11$ \\
\hline NLRP8 & $/$ \\
\hline NLRP9 & $1.62 \mathrm{E}-06$ \\
\hline NLRP10 & $8.69 \mathrm{E}-12$ \\
\hline NLRP11 & $1.53 \mathrm{E}-02$ \\
\hline NLRP12 & $4.92 \mathrm{E}-01$ \\
\hline NLRP13 & $/$ \\
\hline NLRP14 & $8.18 \mathrm{E}-02$ \\
\hline
\end{tabular}

Table 2 Expression of NLRP family (UALCAN) 


\begin{tabular}{|c|c|c|c|c|}
\hline \multicolumn{5}{|l|}{ Comparison } \\
\hline NLRP family & Normal vs Grade 1 & Normal vs Grade 2 & Normal vs Grade 3 & Normal vs Grade 4 \\
\hline NLRP1 & 1.89E-10 & 1.62E-12 & 4.00E-12 & 1.88E-02ه $0.05 \rrbracket$ \\
\hline NLRP2 & 1.88E-03ه $ه 0.05 \rrbracket$ & $1.18 \mathrm{E}-06$ & $3.40 \mathrm{E}-03 \mathbb{\square} \mathrm{0} 0.05 \rrbracket$ & 7.25E-01هष0.05】 \\
\hline NLRP3 & 2.09E-01ه $ه 0.05 \rrbracket$ & 1.70E-03هष0.05】 & 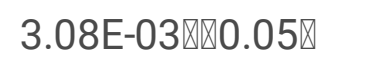 & 2.65E-01هष0.05】 \\
\hline NLRP4 & 3.31E-01ه $ه 0.05 \rrbracket$ & 1.17E-04 & 4.54E-03冈ख0.05】 & 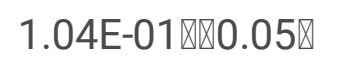 \\
\hline NLRP5 & I & / & / & / \\
\hline NLRP6 & 2.07E-01ه $ه 0.05 \rrbracket$ & $1.32 \mathrm{E}-07$ & 8.01E-08 & 6.17E-02ه凶0.05区 \\
\hline NLRP7 & 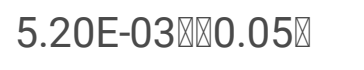 & 4.04E-07 & 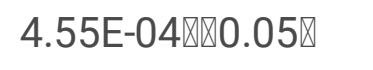 & 3.27E-01ه $₫ 0.05 \rrbracket$ \\
\hline NLRP8 & / & / & / & / \\
\hline NLRP9 & 2.69E-02ه冈0.05》 & 1.03E-04 & 2.35E-05 & 7.94E-02ه■0.05区 \\
\hline NLRP10 & $1.96 \mathrm{E}-06$ & 2.03E-09 & 1.53E-02ه $ه 0.05 \rrbracket$ & 4.43E-01ه $₫ 0.05 \rrbracket$ \\
\hline NLRP11 & 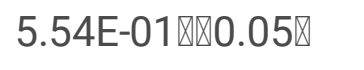 & 7.76E-03ه $₫ 0.05 \rrbracket$ & $1.90 \mathrm{E}-01 \otimes \mathbb{Q} 0.05 \rrbracket$ & 3.34E-01ه $₫ 0.05 \rrbracket$ \\
\hline NLRP12 & 8.40E-01ه $ه 0.05 \rrbracket$ & 3.19E-01هष0.05】 & 9.70E-01《ख0.05区 & 1.65E-01ه $0.05 \rrbracket$ \\
\hline NLRP13 & 4.33E-01ه $ه 0.05 \rrbracket$ & 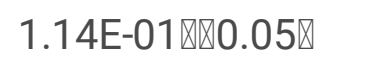 & 2.03E-02ه冈0.05】 & 2.94E-01هष0.05】 \\
\hline NLRP14 & 7.76E-03ه冈0.05》 & 6.62E-02ه $0.05 \rrbracket$ & 5.71E-01ه $ه 0.05 \rrbracket$ & 7.66E-01ه $ه 0.05 \rrbracket$ \\
\hline
\end{tabular}

Table 3 The relationship between the expression of NLRPs and tumor grade

\section{Figures}


A
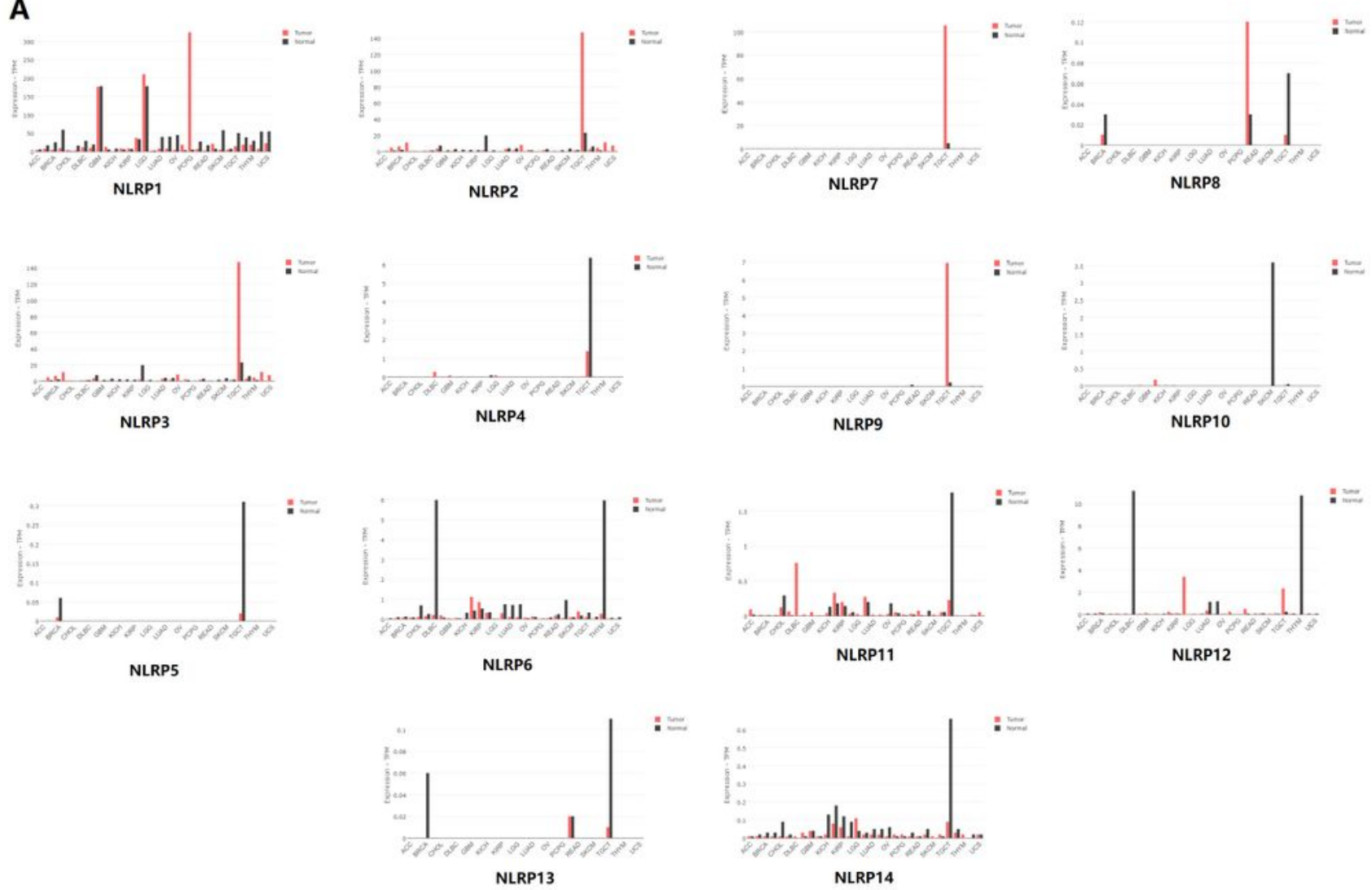

B

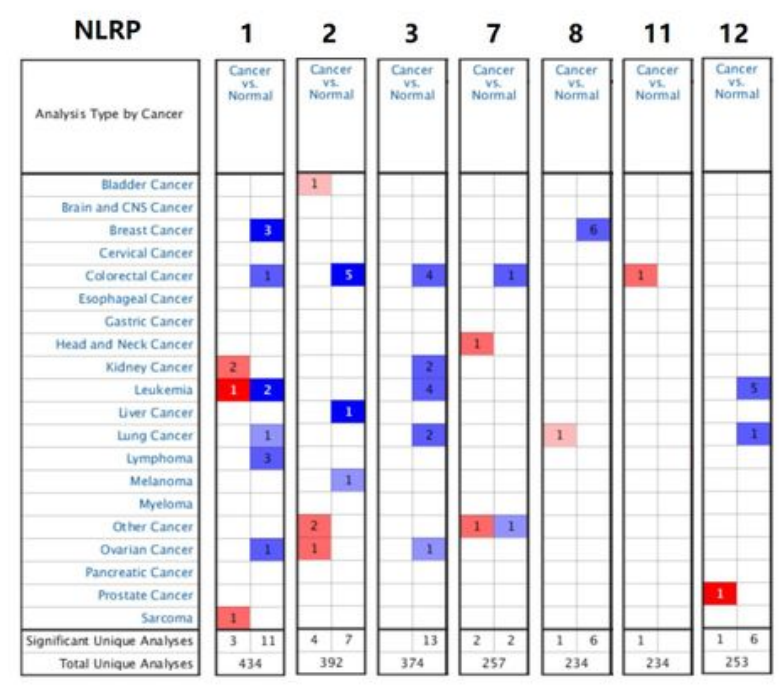

\section{Figure 1}

A.Expression of NLRPs in 31 different types of cancer disease (GEPIA) B. Expression of NLRPs in 20 different types of cancer diseases (Oncomine database). Blue represents decreased expression, red represents increased expression. 

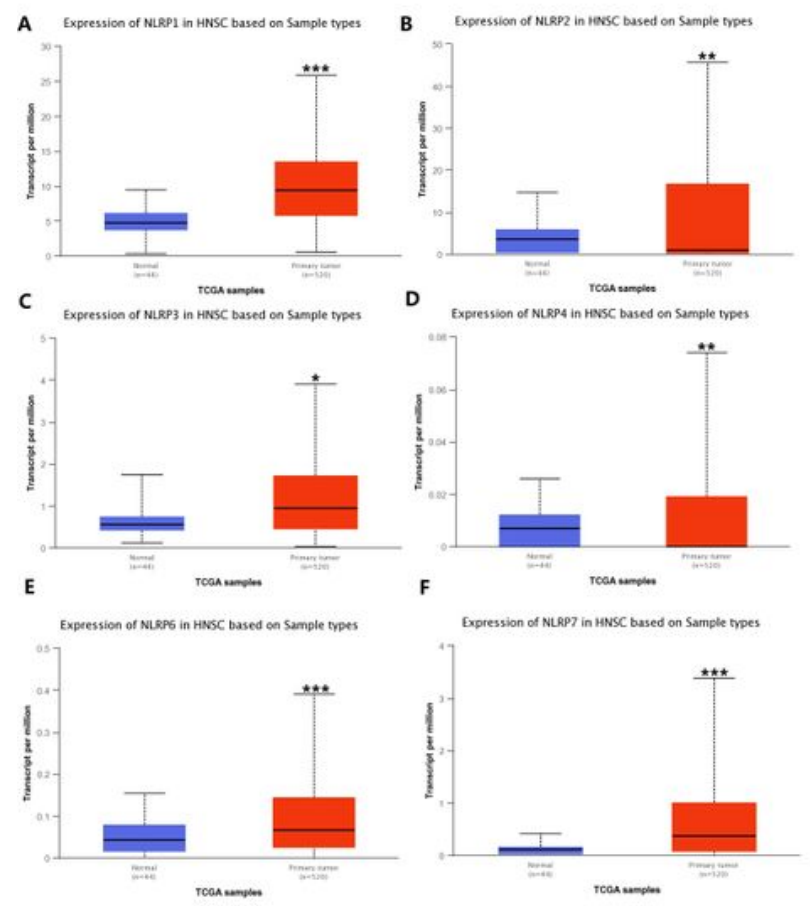

G

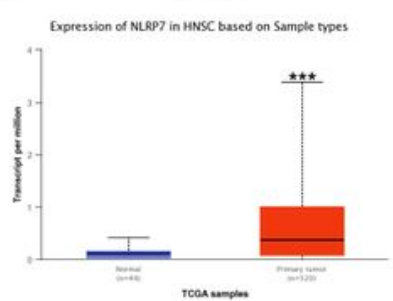

G Expression of MRReg in HNSC based on Sample types

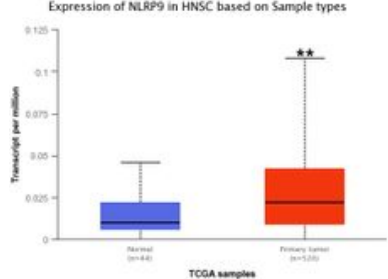

H
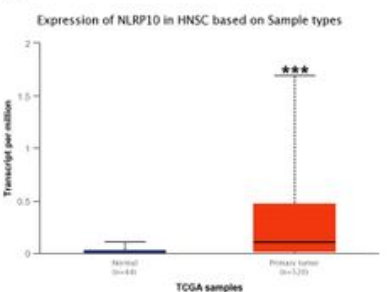

1 Expression of NLRP11 in HinsC based on Sample types
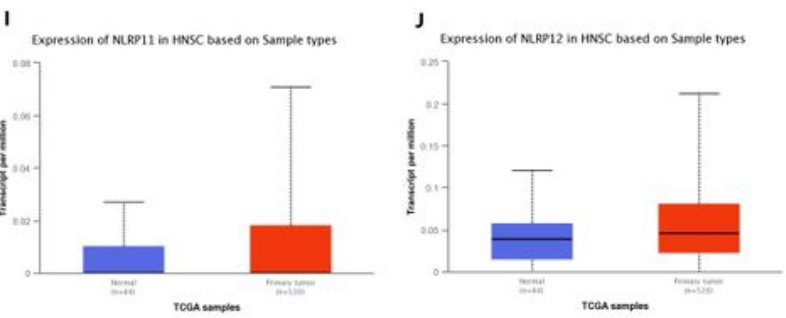

к

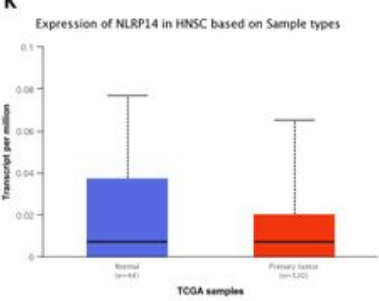

Figure 2

mRNA expression of NLRPs in NPC tissues and adjacent normal tissues (UALCAN). Compared to normal samples, 12 NLRPs were over-expressed in primary HNSCC tissues (A-K:

NLRP1/2/3/4/6/7/8/9/10/11/12/14) 

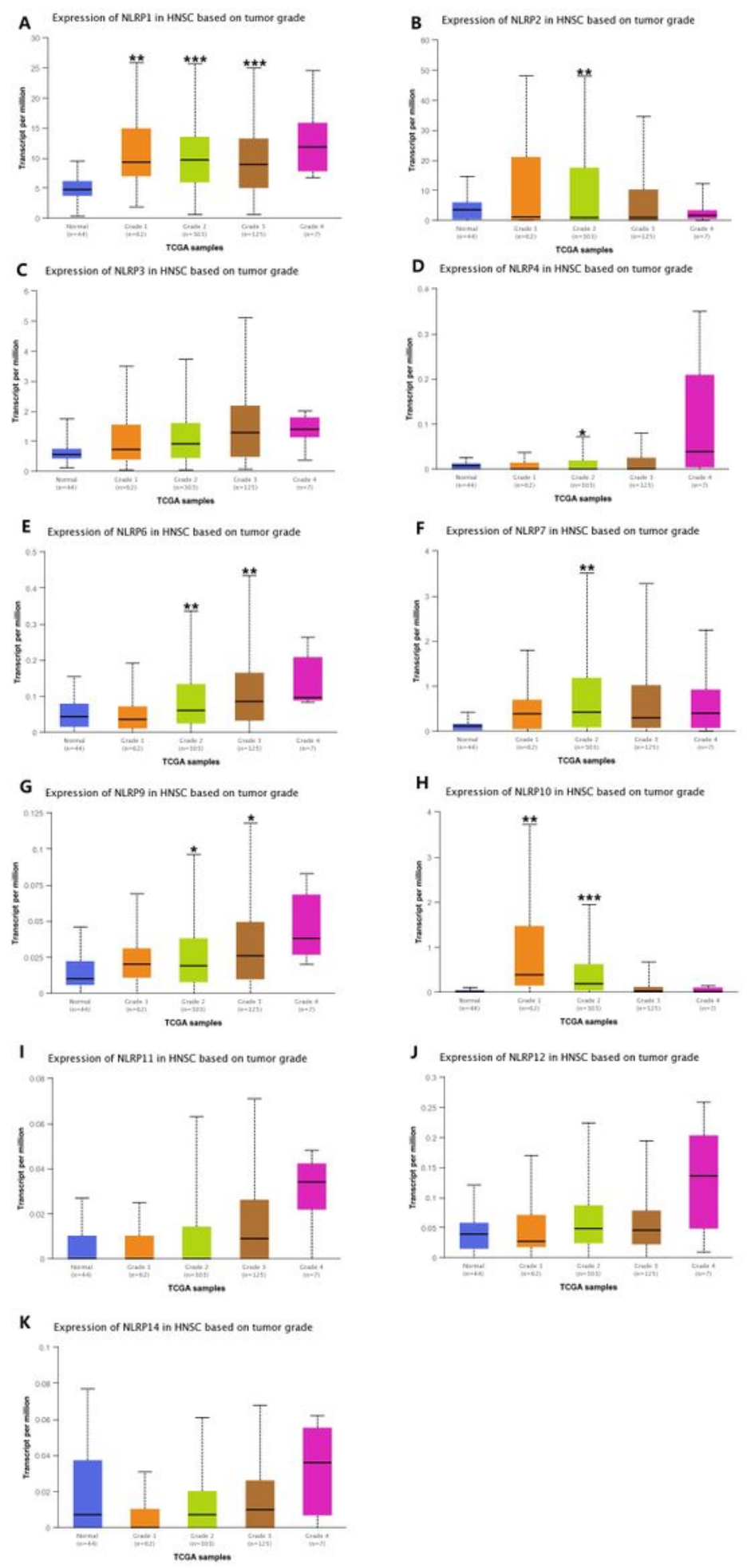

\section{Figure 3}

The relationship between the expression of NLRPs and the clinical manifestations of HNSCC (UALCAN). The highest expression of NLRP 1/2/3/4/6/7/9/11/12/14 mRNAs was found in grade 4 tumors, while the highest expression of NLRP 10 mRNA was found in grade 1 tumors. 


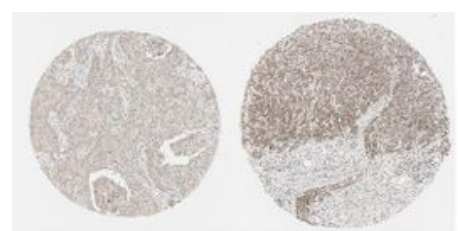

NLRP1

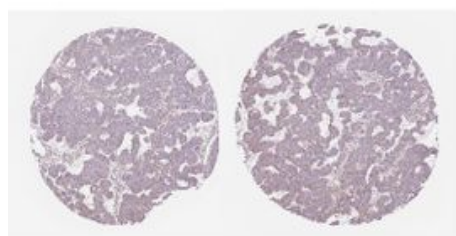

NLRP5

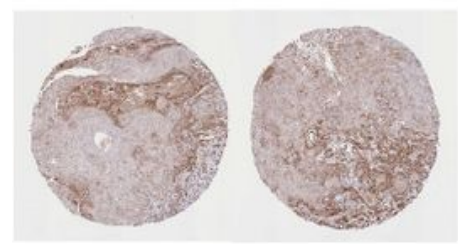

NLRP7

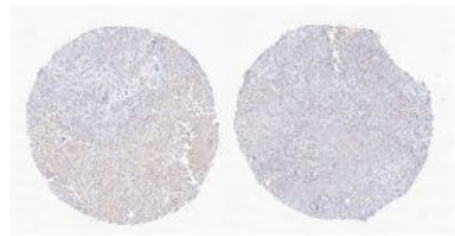

NLRP9

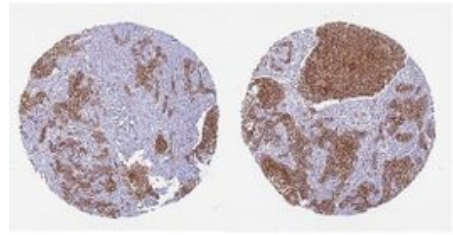

NLRP11

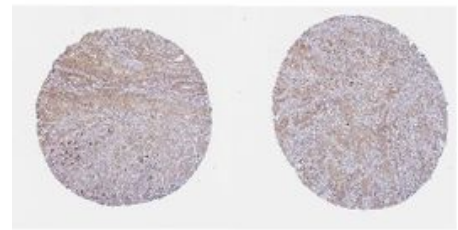

NLRP13

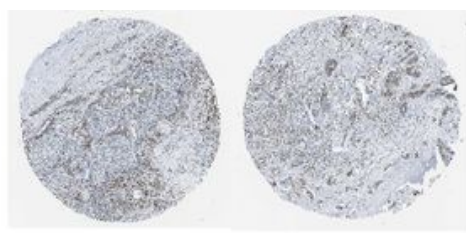

NLRP3

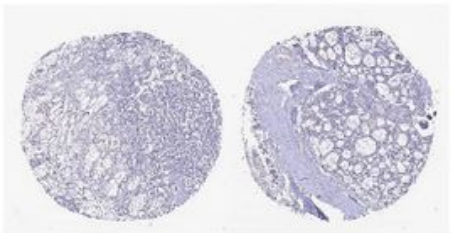

NLRP6

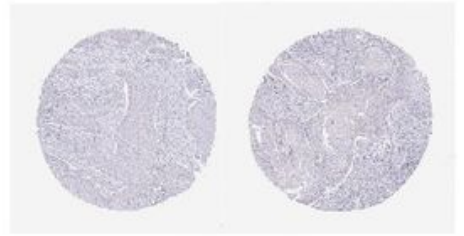

NLRP8

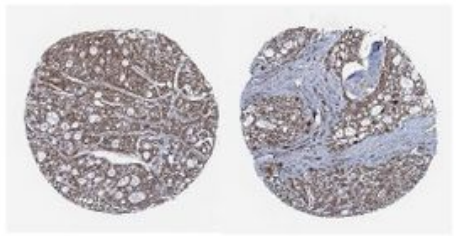

NLRP10

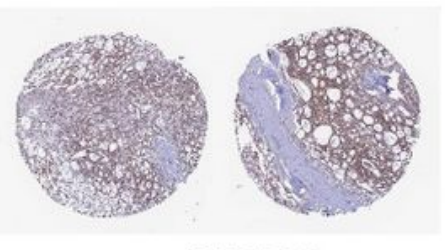

NLRP12

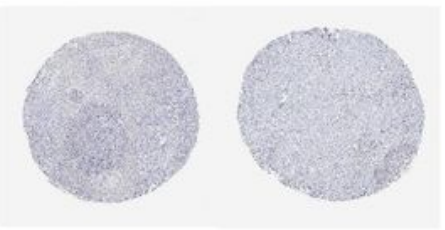

NLRP14

Figure 4

Human Protein Atlas. The mRNA expression levels of NLRP 1/3/5/6/7/8/9//10/11/12/13/14 


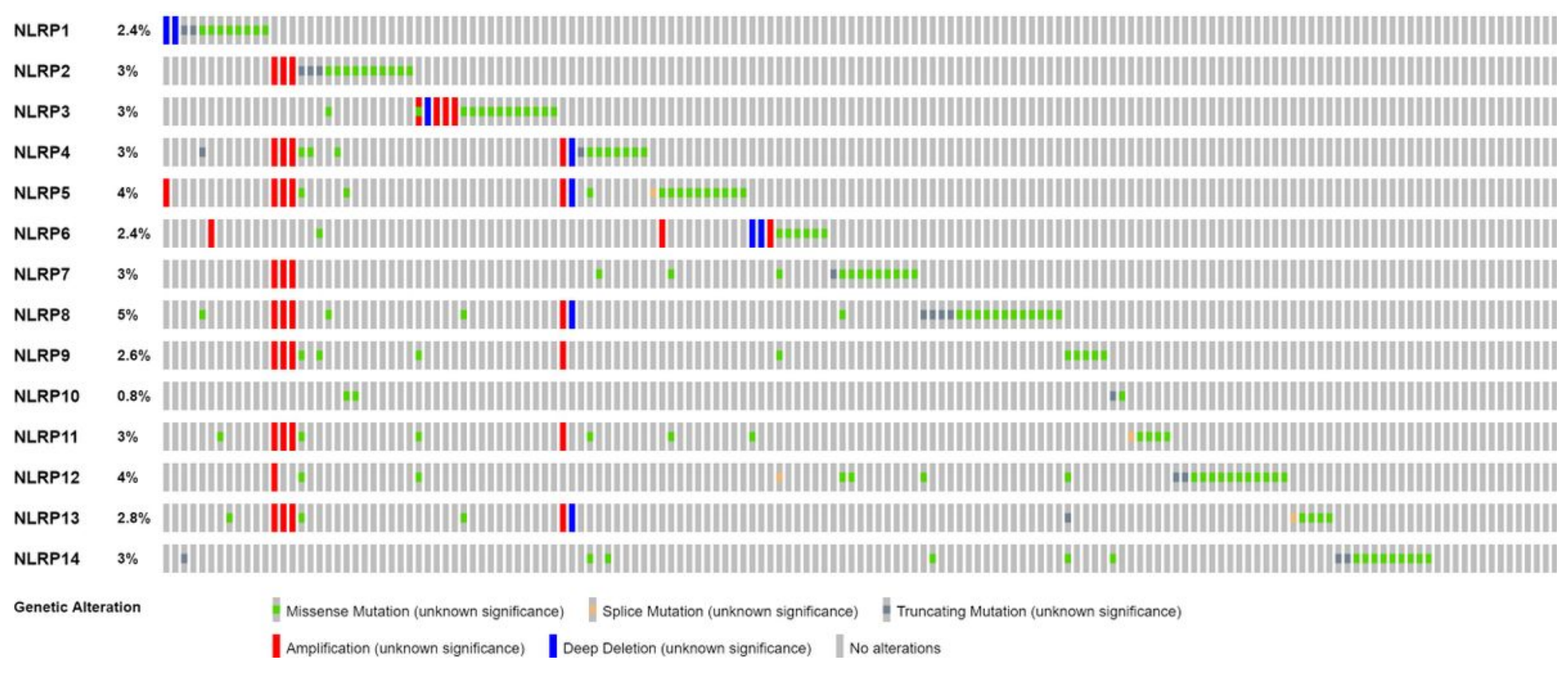

Figure 5

Genetic alteration of NLRPs. NLRP 2, NLRP 3, NLRP 4 ,NLRP 5, NLRP 7, NLRP 8 , NLRP 11 ,NLRP 12 and NLRP13 were genes alterations more than 3\% in HNSCC patients, NLRP 5 is ranked as the highest genes with genetic alterations in HNSCC patients and their mutation rates was $5 \%$. 

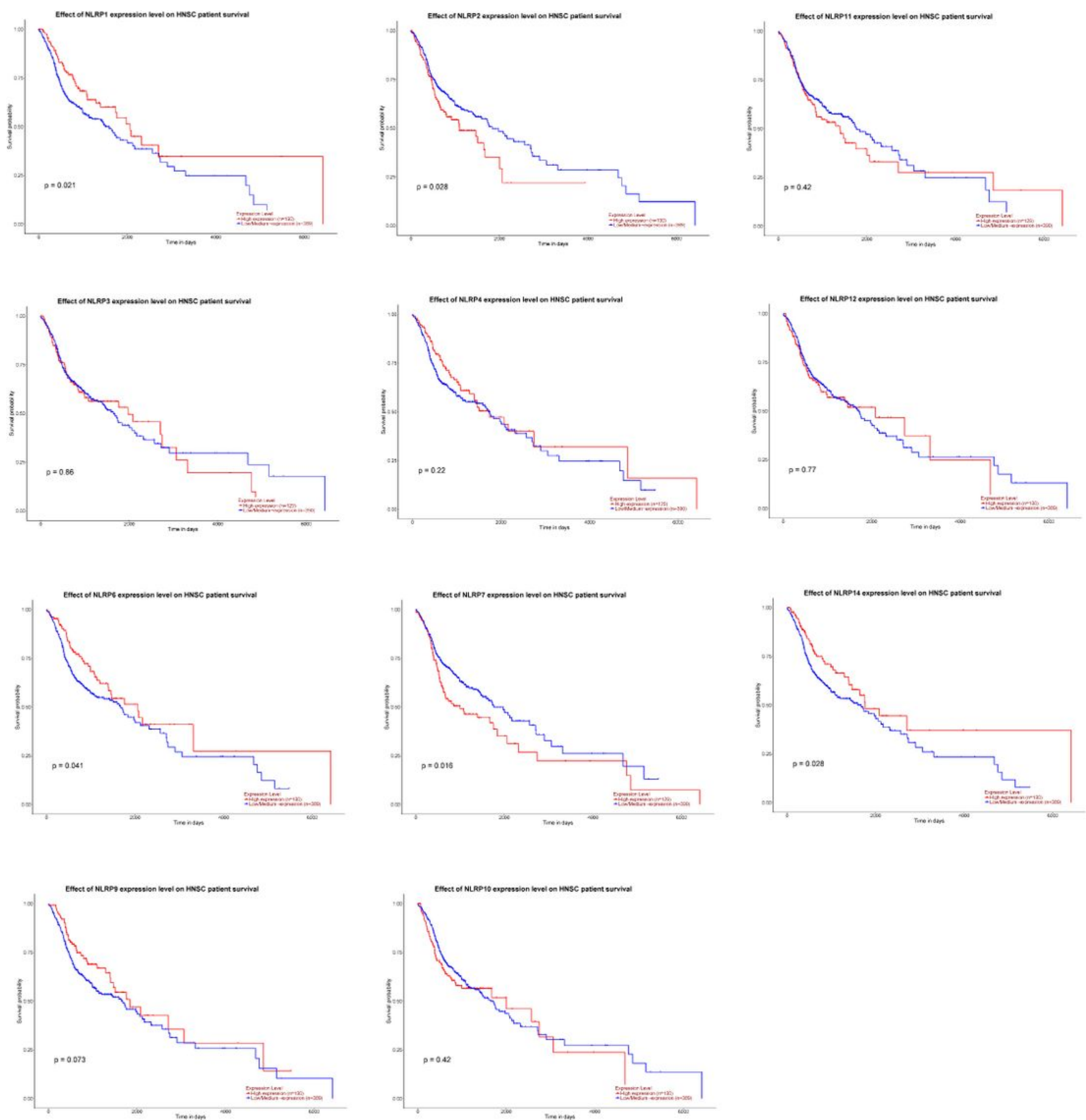

Figure 6

A. Expression of NLRPs associated with the overall survival (OS) of HNSCC patients (UALCAN). Higher expression of NLRPs(1/2/4/6/7/14, p囚0.05) was associated with shorter OS. 

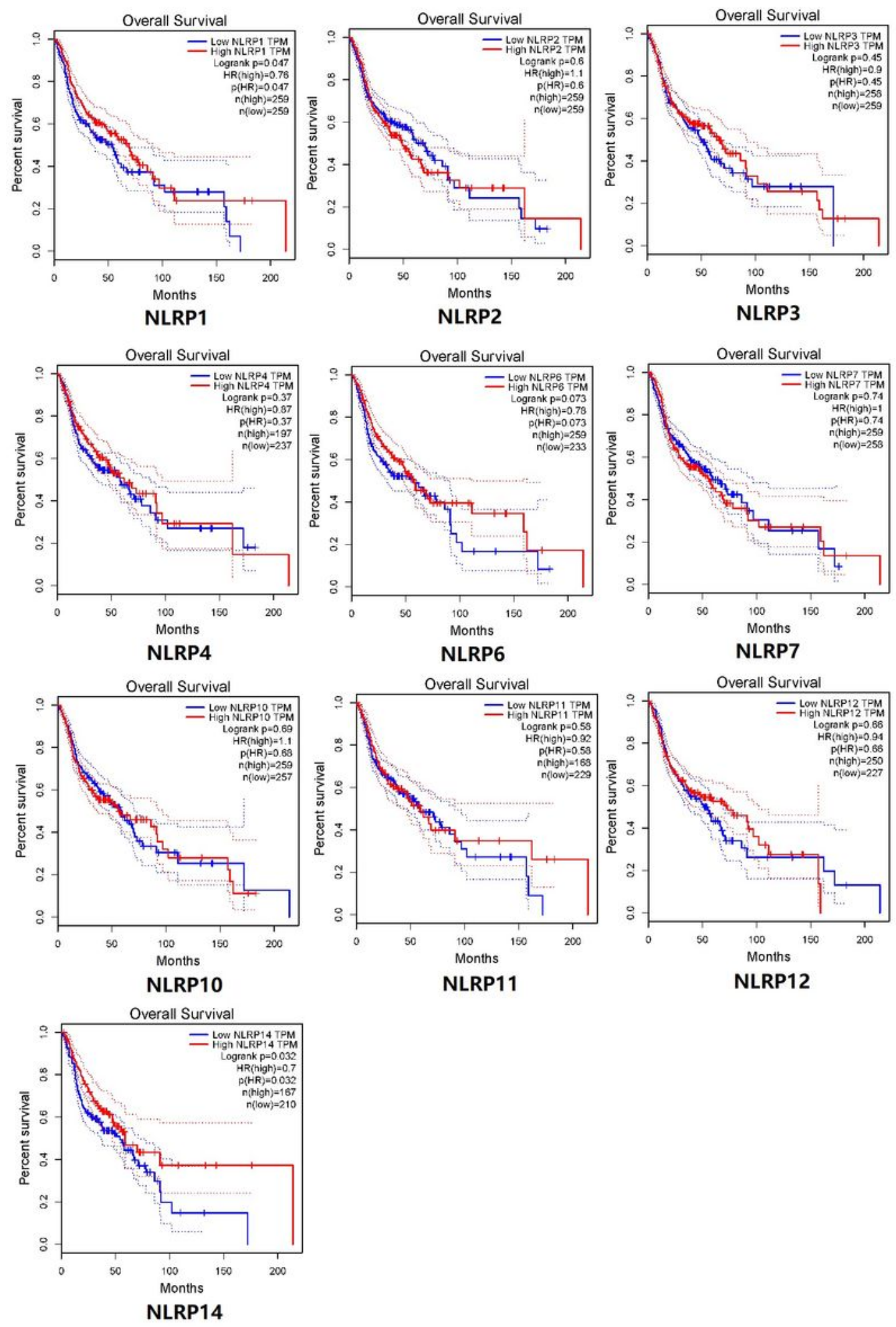

\section{Figure 7}

Prognostic value of expression of distinct NLRPs in HNSCC patients (GEPIA). In general, higher expression of NLRP mRNAs (1/6/14, p $₫ 0.05)$ is related 

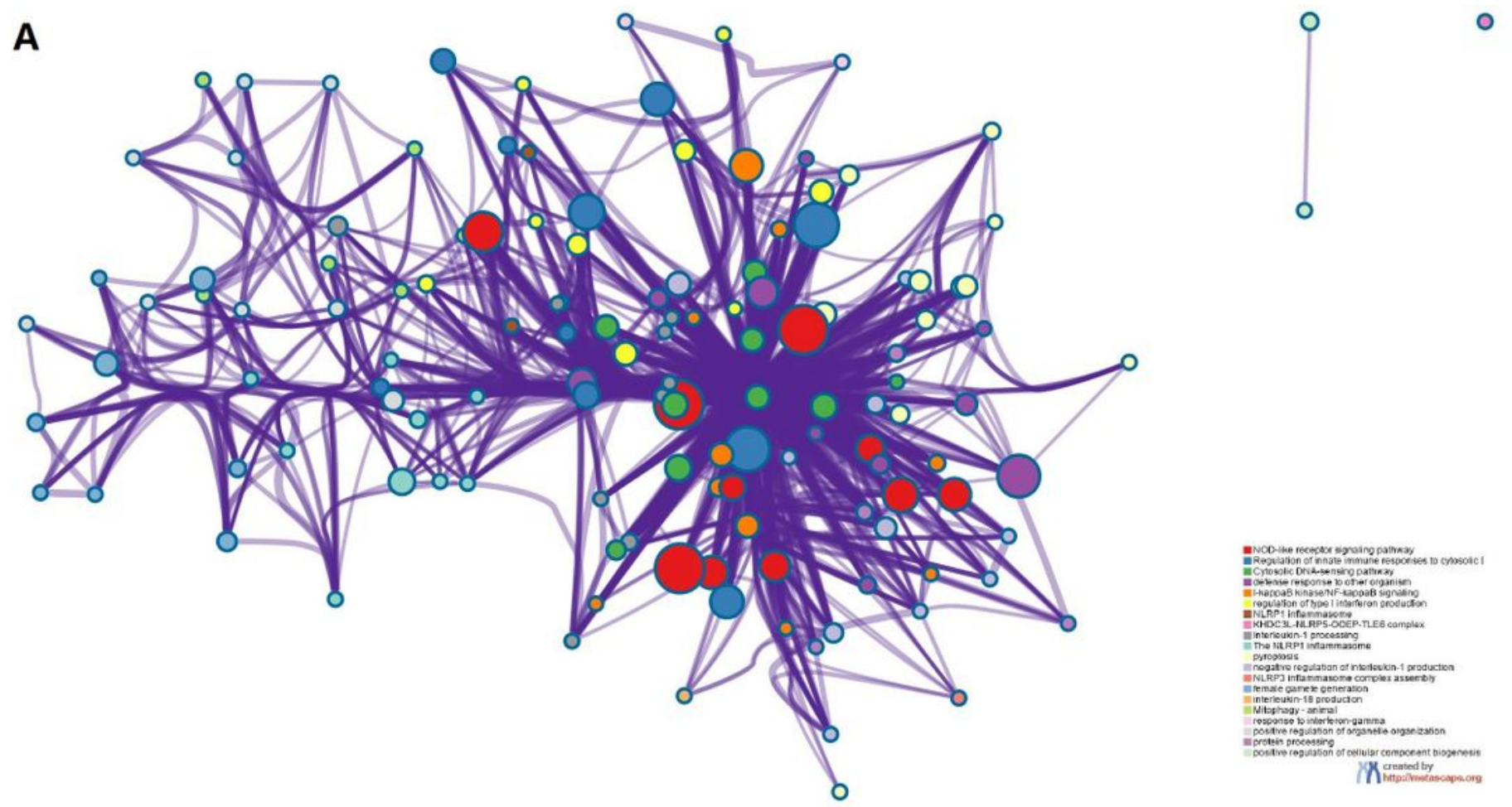

B
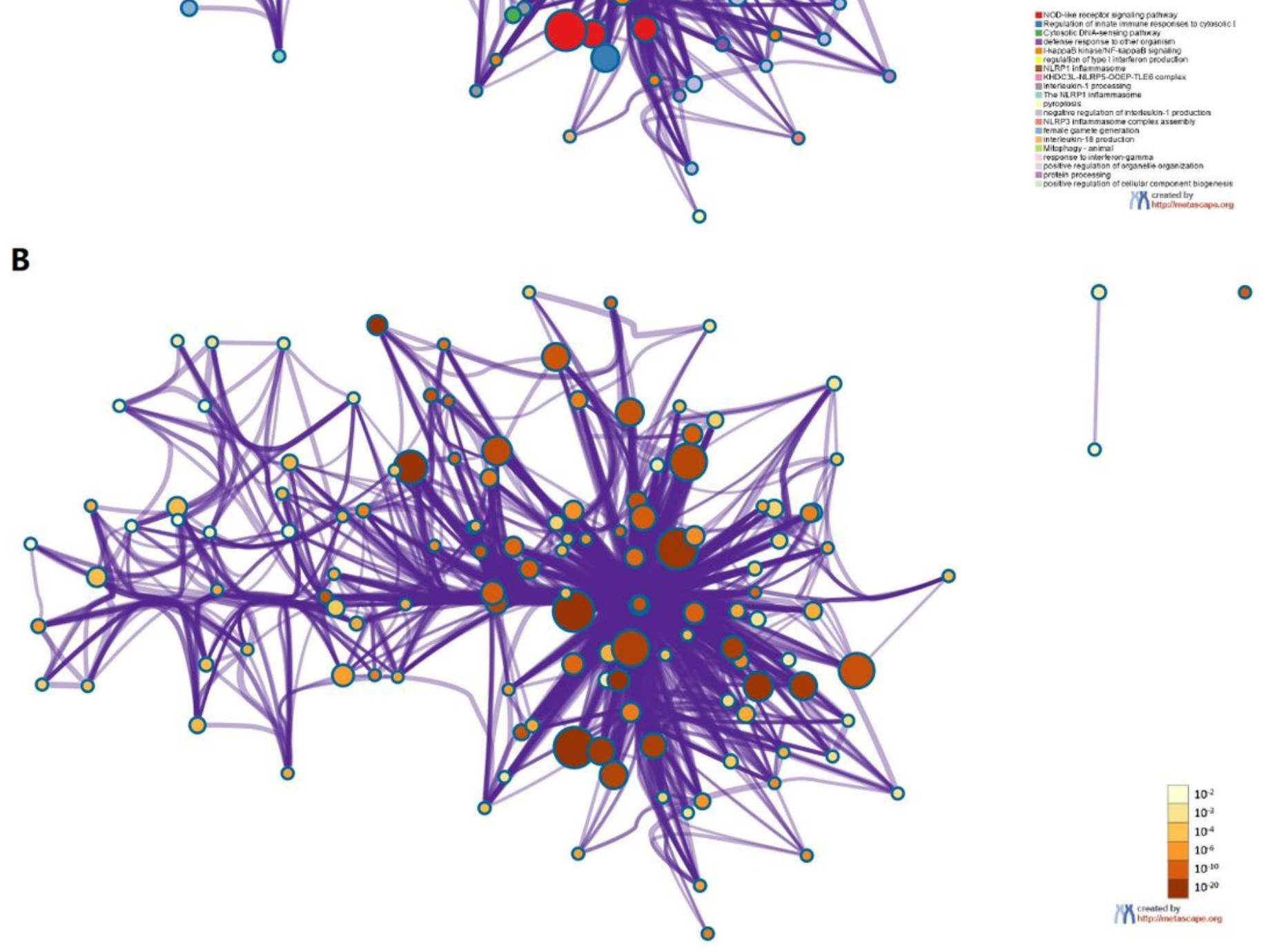

Figure 8

Enriched Ontology Clusters (Colored by Cluster ID and p-Value) of NLRPs and their altered neighbor genes in HNSCC patients (Metascape) 


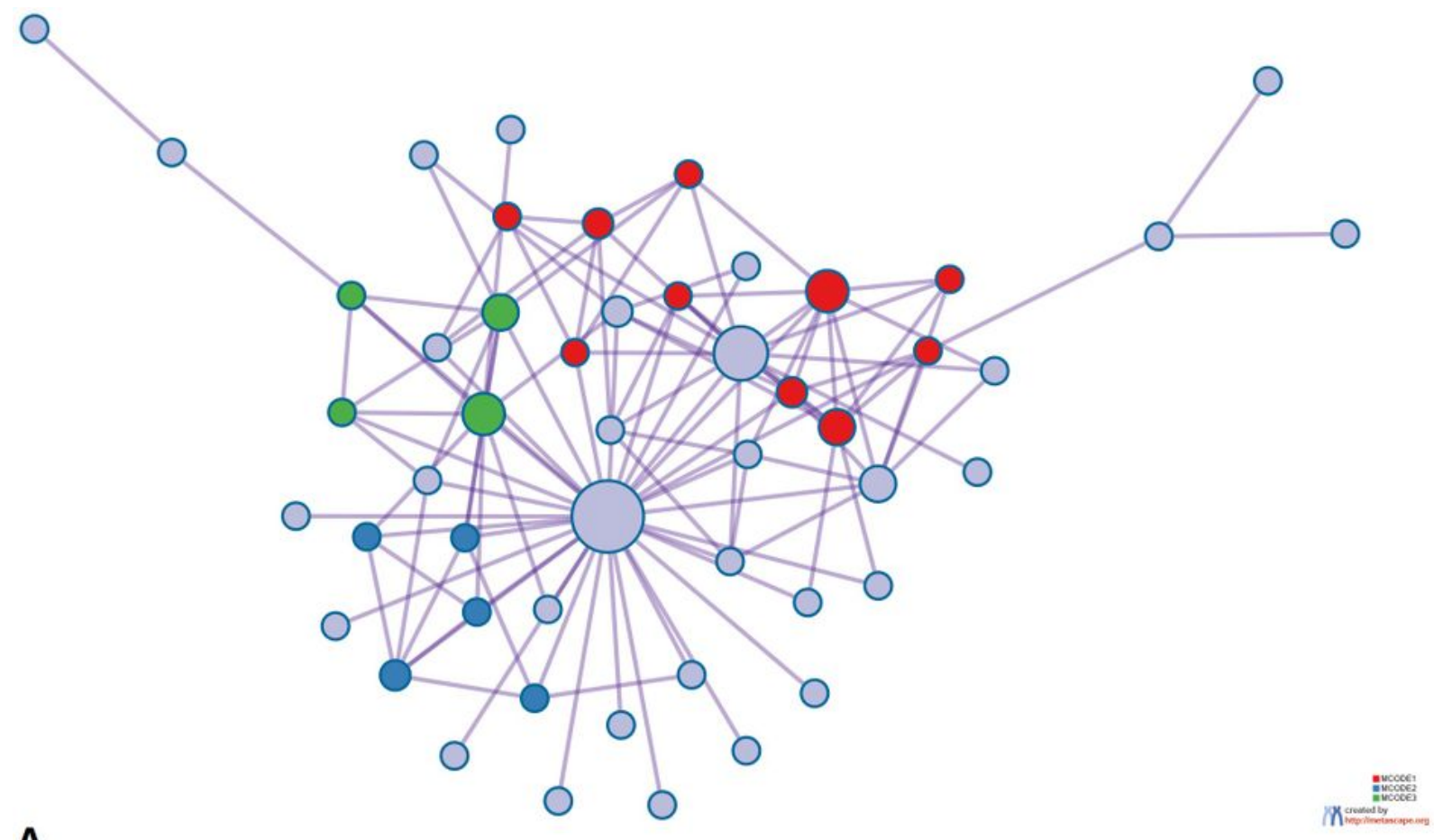

A

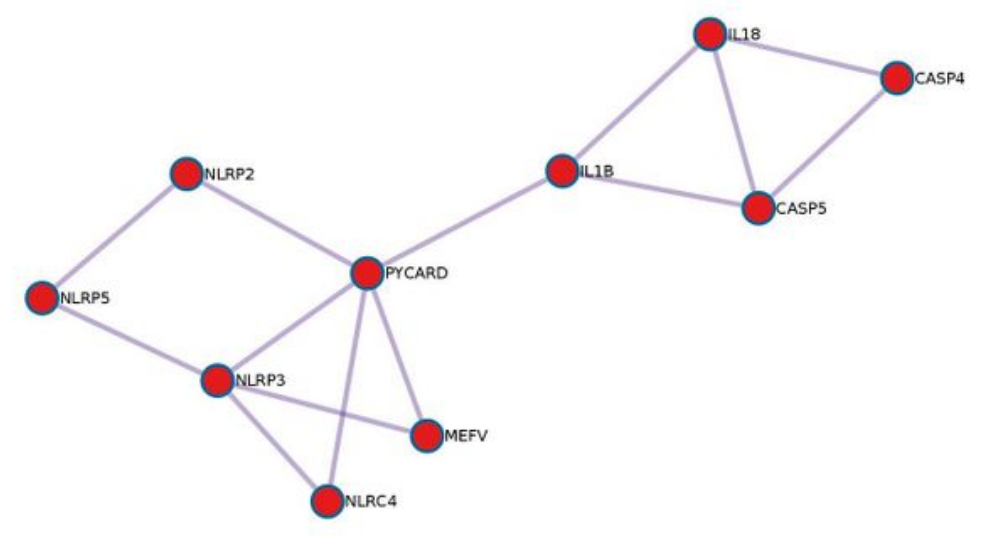

B
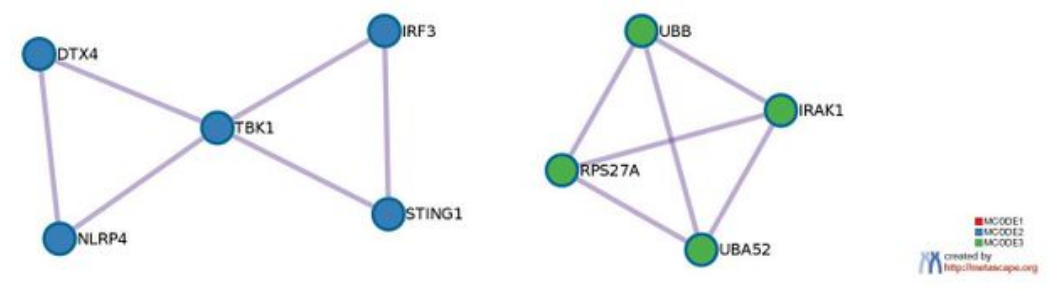

\section{Figure 9}

A. Protein-protein Interaction Network for NLRPs and their altered neighbor genes in HNSCC patients (Metascape) $\triangle \mathrm{B}$. PPI MCODE Components of NLRPs and their altered neighbor genes in HNSCC patients (Metascape) 


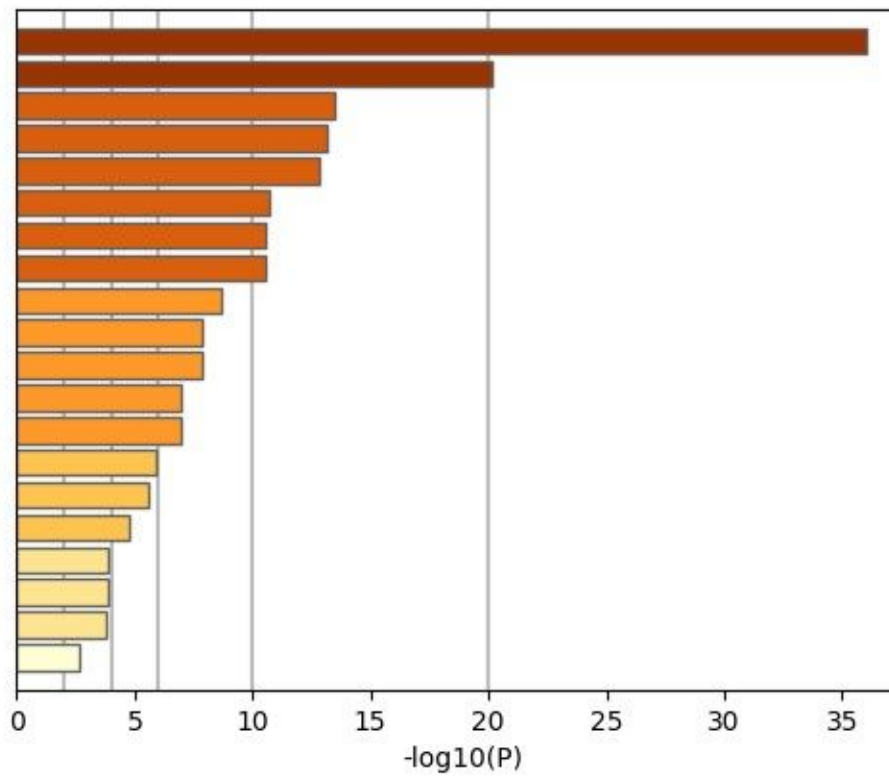

ko04621: NOD-like receptor signaling pathway

R-HSA-3134975: Regulation of innate immune responses to cytosolic DNA ko04623: Cytosolic DNA-sensing pathway

GO:0098542: defense response to other organism

GO:0007249: I-kappaB kinase/NF-kappaB signaling

GO:0032479: regulation of type I interferon production

CORUM:7378: KHDC3L-NLRP5-OOEP-TLE6 complex

CORUM:6317: NLRP1 inflammasome

R-HSA-448706: Interleukin-1 processing

R-HSA-844455: The NLRP1 inflammasome

GO:0070269: pyroptosis

GO:0032692: negative regulation of interleukin-1 production

GO:0044546: NLRP3 inflammasome complex assembly

GO:0007292: female gamete generation

GO:0032621: interleukin-18 production

ko04137: Mitophagy - animal

GO:0034341: response to interferon-gamma

GO:0010638: positive regulation of organelle organization

GO:0016485: protein processing

GO:0044089: positive regulation of cellular component biogenesis

Figure 10

Prediction of functions and pathways of NLRPs and their altered neighbor genes in HNSCC patients (Metascape). Biological processes such as defense response to other organisms, I-kappaB kinase/NFkappaB signaling, regulation of type I interferon production, pyroptosis, negative regulation of interleukin1 production, NLRP3 inflammasome complex assembly, female gamete generation, interleukin-18 production, response to interferon-gamma, positive regulation of organelle organization, protein processing, and positive regulation of cellular component biogenesis. In KEGG analysis, pathways related to the regulation of innate immune responses in cytosolic DNA, interleukin-1 processing, and the NLRP1 inflammasome were associated with the functions of NLRPs. 


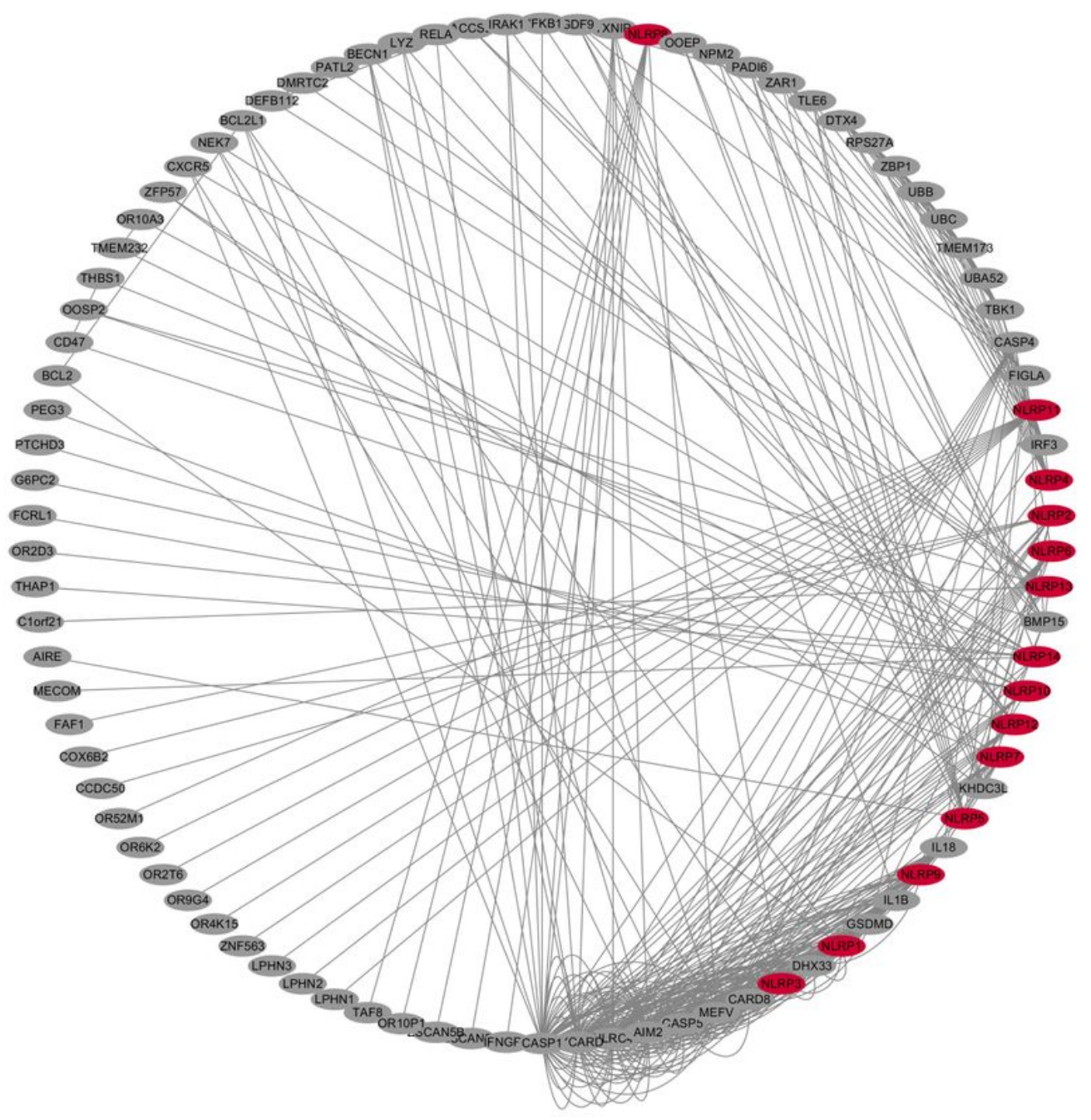

Figure 11

Visualization of NLRPs and their altered neighbor genes in HNSCC patients (String and Cytoscape). NLRPs marked in red. 

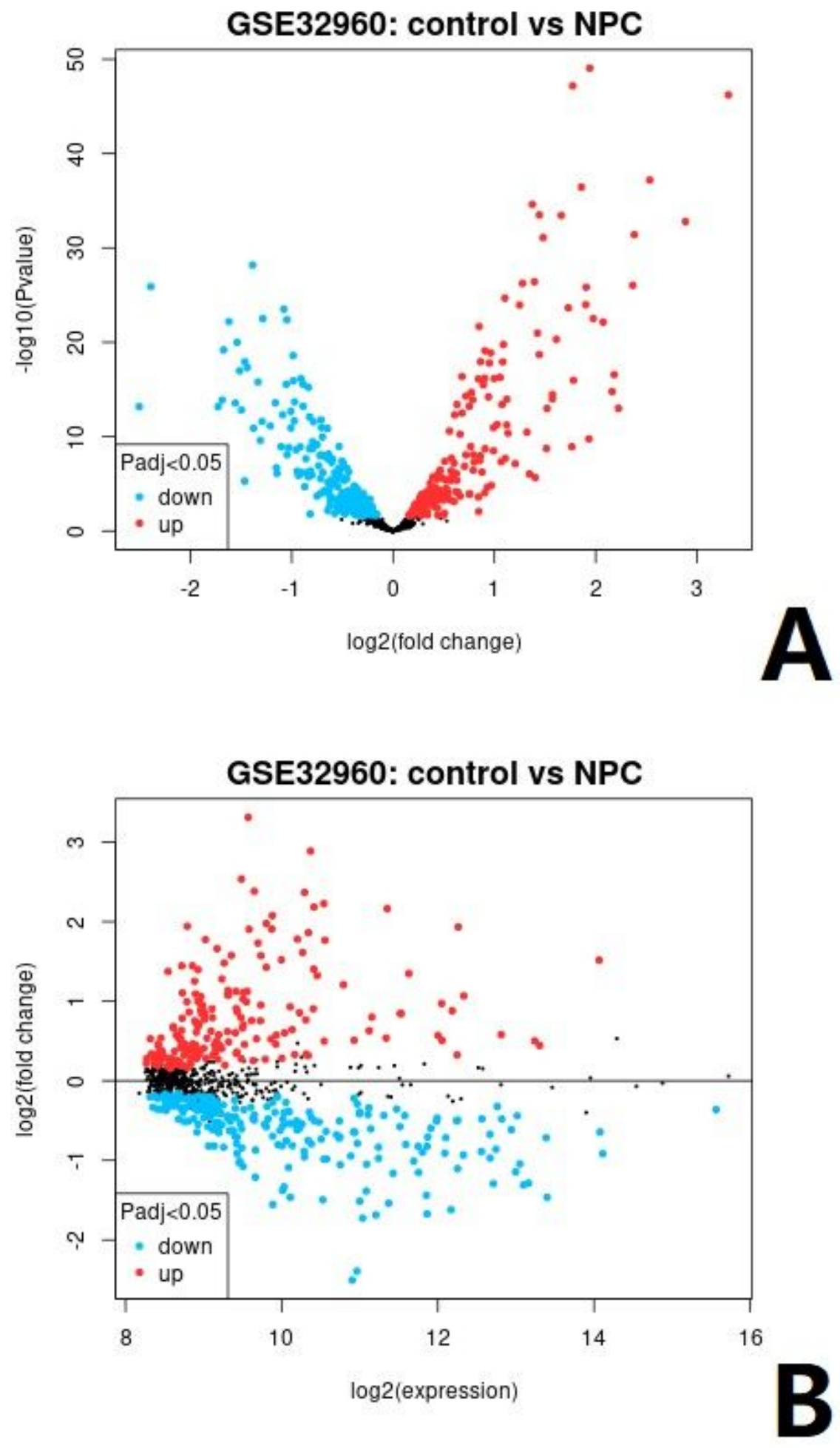

Figure 12

Volcano plot of differentially expressed miRNAs (GEO2R). The red dots represent upregulated miRNAs and the blue dots represent downregulated miRNAs.

\section{Supplementary Files}


This is a list of supplementary files associated with this preprint. Click to download.

- Table1.docx 